\title{
Efecto de la adición de aglomerantes en las propiedades mecánicas de los pellets de biomasa
}

\author{
The addition effect of binders on the durability (PDI) of biomass pellets
}

\author{
Elías Ricardo Durango Padilla ${ }^{1}$ Cristian Berastegui Barranco ${ }^{1}$ Jorge Mario Mendoza Fandiño ${ }^{1 *}$ \\ Recibido 24 de julio de 2017, aceptado 19 de febrero de 2018 \\ Received: July 24, 2017 Accepted: February 19, 2018
}

\begin{abstract}
RESUMEN
La utilización directa de residuos agrícolas y forestales como fuente de energía no es eficiente, debido a que generalmente presentan bajo poder calorífico y baja densidad. La compactación de los residuos puede ser una solución a este problema, una vez que los residuos compactados por ser más densos que los residuos in natura poseen mayor energía por unidad de volumen. Este trabajo tuvo como objetivo evaluar la calidad de pellets fabricados a partir de fibra de coco y residuos de algodón, recolectados en el departamento de Córdoba-Colombia. Fueron elaborados 12 tipos de pellets, mezclando los materiales con tres diferentes tipos de aglomerante (de maíz, yuca y plátano) en dos porcentajes (10\% y 30\%). Otros dos tipos de pellets fueron producidos sin la adición de aglomerantes. Después de dos semanas, se determinó el índice de durabilidad (PDI) para todos los tratamientos de acuerdo con la norma estándar ASABE 269.4. También se determinó el contenido de humedad y tamaño de partículas en los materiales. El PDI de pellets elaborados sin aglomerantes fue comparado con el PDI de pellets con aglomerantes, los resultados mostraron que ambos pellets tuvieron PDI superior a 97,95\% y estadísticamente no hubo diferencias significativas al variar el tipo de aglomerante. Por tanto, fue posible la producción de pellets con buena calidad sin uso de aglomerantes, utilizando residuos de coco y algodón con tamaños de partícula inferiores a $2 \mathrm{~mm}(10 \mathrm{mesh})$ y contenido de humedad entre 10 y $15 \%$.
\end{abstract}

Palabras clave: Combustible sólido, residuos, resistencia mecánica.

\begin{abstract}
The direct use of agricultural and forestry waste as energy source is not efficient since they generally have low calorific value and density, the resides compaction can be a solution to those problems. The compacted residues are denser than the unprocessed waste, because of that, it has higher energy per volume unit. The objective of this work was to evaluate the quality of pellets manufactured from coconut fiber and cotton residues; both collected in the state of Córdoba-Colombia. It was made twelve experimental pellets, testing three different binders (corn, cassava and banana) in two different percentages (10 and $30 \%$ ) and two types of pellets were made without the addition of any binders. After two weeks, the PDI (durability) for all treatments was determined according to the ASABE 269.4 standard. The moisture content and particle size in the materials were also determined. The PDI of pellets produced without binders was compared with the PDI of pellets with binders. The results showed that both pellets had PDI higher than $97.95 \%$ and statistically there were no significant differences when the type of binder varied. It was therefore, possible to produce pellets with good quality without the use of binders, using
\end{abstract}

\footnotetext{
1 Departamento Ingeniería Mecánica. Universidad de Córdoba. Carrera 6 \# 76-103. Montería, Colombia. E- mail: erdurango22@gmail.com; cberasb@gmail.com; jorge.mendoza@correo.unicordoba.edu.co

Autor de correspondencia
} 
coconut fiber and cotton residues only, with particle sizes of less than $2 \mathrm{~mm}$ (10 mesh) and moisture content between 10 and $15 \%$.

Key words: Solid fuel, waste, mechanical strength.

\section{INTRODUCCIÓN}

La producción de pellets se inició en América del Norte en la segunda mitad de la década de 1970 y más tarde se extendió a Europa y otras partes del mundo [1]. Hoy en día, los pellets se producen y utilizan a escala mundial, pero su uso se concentra en los países desarrollados. La densificación es un proceso por el cual partículas sueltas, tales como aserrín o residuos agroindustriales triturados se comprimen mecánicamente con o sin el uso de un aglomerante para formar productos más compactos, llamados pellets o briquetas [2].

Esta técnica permite aumentar la densidad de los residuos mejorando su manipulación y transporte, por otro lado, con el aumento de la densidad aumenta también la energía por unidad de volumen [3], por lo tanto, en los últimos años la densificación de residuos viene siendo utilizada principalmente en los países en desarrollo para aprovechar de forma más eficiente residuos agrícolas y forestes como fuente de energía [2, 4-6]. La mayoría de la biomasa densificada producida en países en desarrollo tiene forma de briquetas y se utilizan directamente para sustituir la leña o el carbón [7].

El ensayo de durabilidad es un método utilizado por la industria para medir y controlar la calidad de pellets y briquetas, estos productos tienden a quebrarse cuando son manipulados, el ensayo simula ese proceso y prevé el porcentaje de finos generados, conocido como índice de durabilidad. Por tanto, la durabilidad puede ser interpretada como la capacidad de pellets y briquetas para resistir la manipulación y el transporte [8]. Investigadores como Kaliyan y Morey encontraron que el tamaño de partícula, el contenido de humedad en la materia prima y el precalentamiento durante la densificación afecta la densidad y durabilidad de los pellets [9].

Kaliyan y Morey definen el índice de durabilidad o resistencia a la abrasión como la relación entre la masa que queda después de separadas las partículas finas producidas durante el ensayo sobre la muestra total ensayada [9]. Existen normas estandarizadas para calcular el índice de durabilidad (PDI), o simplemente porcentaje de durabilidad, el procedimiento más usado en la industria de los Estados Unidos se detalla en la norma estándar de la sociedad americana de ingenieros agrícolas y biológicos ASABE 269.4 [10]. Sin embargo, otras normas también son ampliamente utilizadas en diversos estudios [11-13].

Grandes cantidades de desechos agrícolas se generan en las actividades agrícolas y en las industrias de procesamiento de productos agrícolas, en el departamento de Córdoba-Colombia la economía se basa principalmente en actividades ganaderas y agrícolas, según el Departamento Administrativo Nacional de Estadística, para el año de 2014 se registró una producción de 68.596 tonelada de algodón y de 29.949 toneladas de coco en el año de 2013 [14-15]. En este contexto el objetivo del presenté trabajo fue producir pellets a partir de residuos de coco y algodón, mezclados con diferentes concentraciones de aglomerantes (de maíz, yuca y plátano) y evaluar la durabilidad de los pellets usando la norma estándar ASABE 269.4.

\section{MATERIAL Y MÉTODOS}

Los residuos de coco y algodón, fueron recolectados en las vías que comunican a la ciudad de Montería con Cereté y en las instalaciones de la Universidad de Córdoba-Colombia, respectivamente. Inicialmente, fue determinado el contenido de humedad de los materiales utilizando una estufa con circulación de aire a $110^{\circ} \mathrm{C}$, luego los materiales fueron triturados y realizada la clasificación de partículas utilizando 100 gramos de cada material triturado y seco, siguiendo el método de tamices estandarizados y la norma ASTM E11 [16]. Durante el procedimiento se usaron tamices de 20, 40, 60 y 100 mesh y una tamizadora marca D\&R, RF SL-305S para agitar la muestra durante $15 \mathrm{~min}$. 
Preparación de las mezclas y fabricación de Pellets En la Tabla 1 se muestra el arreglo experimental que fue seguido para elaborar las mezclas. Éste fue desarrolló basado en un modelo de mezclas combinado con factores categóricos utilizando el programa Design Expert 9, en su versión de prueba.

Tabla 1. Arreglo experimental.

\begin{tabular}{ccccc}
\hline \multicolumn{2}{c}{ Ref. Pellets } & $\begin{array}{c}\text { Biomasa } \\
(\%)\end{array}$ & $\begin{array}{c}\text { Aglomerante } \\
(\%)\end{array}$ & $\begin{array}{c}\text { Tipo de } \\
\text { aglomerante }\end{array}$ \\
\hline R1 & E1 & 90 & 10 & Yuca \\
R2 & E2 & 70 & 30 & Yuca \\
R3 & E3 & 90 & 10 & Plátano \\
R4 & E4 & 70 & 30 & Plátano \\
R5 & E5 & 90 & 10 & Maíz \\
R6 & E6 & 70 & 30 & Maíz \\
R7 & E7 & 100 & 0 & - \\
\hline
\end{tabular}

Pellets de fibra de coco Ref R, pellets de residuos de algodón Ref. E.

Fuente: Elaboración propia.

Siguiendo el orden de la Tabla 1 se mezclaron manualmente la fibra de coco y el tipo de aglomerante correspondiente (maíz, yuca o Plátano) según los porcentajes indicados en el arreglo experimental. Repitiendo el mismo procedimiento se mezclaron por aparte los residuos de algodón con los tres tipos de aglomerante.

Una vez preparadas las mezclas se procedió entonces a fabricar los 12 tipos de pellets con aglomerante, utilizando una pelletizadora tipo rodillo marca Gemco modelo ZLSP D- 200B equipada con una matriz plana perforada. Cada tratamiento después de mezclado fue suministrado a la pelletizadora por la tolva, los rodillos de la máquina presionan el material contra la matriz que se mantiene en movimiento logrando así la producción continua de los pellets con diámetro nominal de $4 \mathrm{~mm}$. Finalmente cada tipo de pellets fue identificado por una referencia, secados al sol durante tres días y almacenados en sacos plásticos.

Siguiendo el mismo procedimiento de fabricación, también fueron elaboraron por separado otros dos tipos de pellets, compuestos por $100 \%$ fibra de coco y $100 \%$ residuo de algodón sin adición de aglomerantes (R7 y E7, respectivamente).

\section{Ensayo de durabilidad}

Para evaluar la calidad de los pellets fabricados se utilizó el ensayo de durabilidad. Se determinó el
PDI en todos los tipos de pellets, con el propósito de comparar los resultados entre sí y verificar la influencia de los aglomerantes en está propiedad mecánica.

El ensayo de durabilidad fue realizado con la adaptación de la norma estándar ASABE 269.4, utilizando un prototipo friabilómetro, equipado con una caja rectangular de dimensiones interiores de $30,5 \times 30,5 \times 12,7 \mathrm{~cm}$ y un motor controlado por PLC. Primero fue pesada una muestra de $250 \mathrm{~g}$ de pellets, esta se colocó dentro de la caja del equipo y sometida a volteó durante 10 minutos a $50 \mathrm{rpm}$. $\mathrm{Al}$ terminar un total de 500 vueltas se separaron los finos generados utilizando un tamiz con abertura 0.8 veces el diámetro de los pellets $(3,2 \mathrm{~mm}$ para este caso) y finalmente se calculó el PDI en porcentaje como la relación entre la masa retenida en el tamiz y la masa total utilizada en el ensayo [10].

\section{Análisis estadístico}

Los análisis estadísticos fueron realizados en el programa Statgraphics versión 2.15.05. El procedimiento comparó los datos obtenidos, mediante análisis de homogeneidad Bartlett, varianza ANOVA y Tukey. La prueba-F en la tabla ANOVA determinó si hubo diferencias significativas entre las medias, en el caso positivo se procedió con las Pruebas de Rangos Múltiples con el fin de analizar cuáles medias eran significativamente diferentes de las otras. Para todos los análisis fue considerado un nivel de confianza de 95\% $(\alpha=0,05)$ [17].

\section{RESULTADOS}

\section{Contenido de humedad y granulometría}

Los resultados de este estudio mostraron que el contenido de humedad para la fibra de coco y los residuos de algodón fueron 13,12 y 10,78\%, respectivamente. El contenido de humedad necesario para peletización depende de la tecnología y la materia prima utilizada [18], los efectos del contenido de humedad en la peletización y la calidad de pellets, ha sido el objetivo de varios estudios, en general el contenido de humedad adecuado para fabricar pellets varía entre $5 \%$ a $10 \%$ para residuos de madera, mientras que para residuos agrícolas varía entre $10 \%$ a 20\% [19-22]. Por lo tanto, se puede decir que los materiales estudiados contenían humedad en el rango recomendando por la literatura. 
Los tamaños de partículas adecuados para la producción de pellets, también dependen del proceso de densificación utilizado, estudios indican que la fricción entre la matriz y los rodillos de las peletizadora aumenta con la disminución del tamaño de partículas, y sugieren utilizar tamaños inferiores a $5 \mathrm{~mm}$, sin embargo, partículas finas (menores a $0,5 \mathrm{~mm}$ ) necesitarían de un agente aglomerante para mejorar la calidad de los pellets [9, 20, 23].

Los materiales después de la trituración mostraron tamaños adecuados para la producción de pellets debido a que la peletizadora utilizada sugería usar tamaños inferiores a $4 \mathrm{~mm}$. La clasificación granulométrica se muestra en la Figura 1.

Fuente: Elaboración Propia.

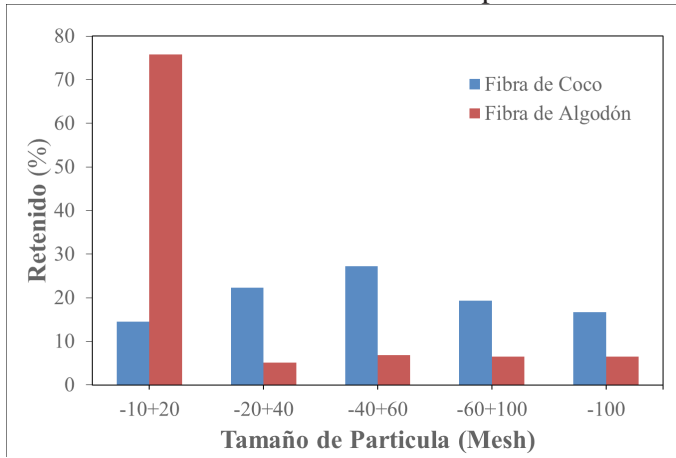

Figura 1. Clasificación granulométrica de los materiales.

En la Figura 1. se puede observar que la mayor fracción de los residuos de algodón triturado quedó retenida en el tamiz número 20 mesh, lo que indica que el 76\% de las partículas de este residuo presentaron tamaños entre 2 y $0.85 \mathrm{~mm}$. Por otro lado, la fibra de coco presentó una distribución de tamaño más uniformemente, con la mayor fracción retenida en el tamiz número 60 mesh.

\section{Índice de durabilidad}

La Tabla 2, muestra los resultados del PDI, para pellets elaborados sin aglomerantes.

Tabla 2. PDI para pellets de biomasa sin aglomerante

\begin{tabular}{|c|c|}
\hline Ref. Pellets & PDI \\
\hline R7 & $98,27 \pm 0,07$ \\
\hline E7 & $97,99 \pm 0,51$ \\
\hline
\end{tabular}

Fuente: Elaboración Propia.
La Figura 2 muestra los resultados de PDI para pellets con aglomerantes.

Fuente: Elaboración Propia.

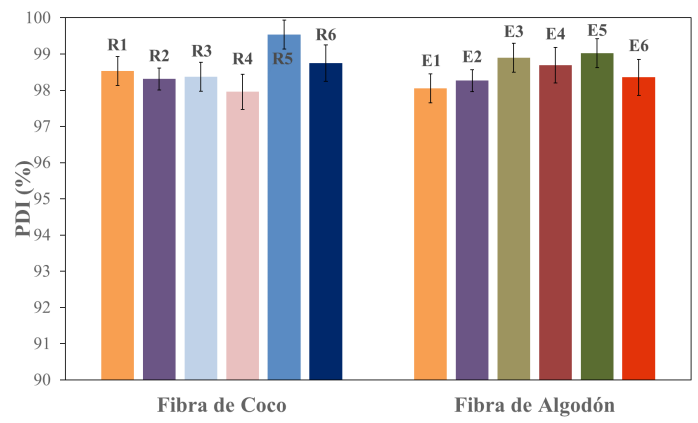

Figura 2. PDI para pellets de biomasa con aglomerante.

Comparando el PDI de los pellets de fibra de coco con aglomerantes y el PDI de R7, el aumento más representativo de la durabilidad fue de $1,27 \%$ utilizando el aglomerante de maíz (tratamiento R5). Similarmente al comparar el PDI de los pellets de algodón con aglomerantes y el PDI de E7, el aumento fue de 1,03\% también al utilizar 10\% de aglomerante de maíz (tratamiento E5).

La durabilidad es uno de los parámetros más importantes en la producción de pellets, de acuerdo con la norma prEN 14961-2 el valor mínimo para el PDI es de 97,5\% para las clases A1 y A2 [18]. Los resultados fueron superiores a el valor mínimo y similares a los encontrados en la literatura, Arzola encontró valores medios de PDI entre $91,02 \%$ y 98,91\% para pellets de cuesco de palma de aceite misturados con melaza como aglomerante en porcentajes entre $15 \%$ y $25 \%$ [24]. Filbakk produjo pellets de madera con PDI entre $92,0 \%$ y $96,0 \%$ y humedad entre $7,0 \%$ y $12,5 \%$ [21].

A partir de los resultados se puede afirmar que no se presentaron aumentos significativos en el PDI que justifiquen la pelletización utilizando los tipos de aglomerantes estudiados. Fue posible fabricar pellets usando fibra de coco o residuos de algodón con buena calidad sin adicionar ningún tipo de aglomerante. La biomasa por sí sola, puede comportarse como una aglomerante ya que por lo general tiene estructura fibrosa que contiene componentes oleosos o pegajosos que facilitan la densificación y mejoran las propiedades de los combustibles densificados [25]. La lignina presente 
en la materia prima a temperaturas elevadas actúa como aglomerante [9], posiblemente la fricción en el proceso de pelletización aumentó la temperatura de la biomasa, lo cual mejoró el proceso de densificación.

La prueba estadística ANOVA permitió confirmar que no hubo una diferencia estadísticamente significativa con un nivel de confianza del 95,0\% entre las medias de los datos, esto significa que no hubo diferencia significativa en el PDI al cambiar de aglomerante usando fibra de coco o residuos de algodón como biomasa. El estadístico de Bartlett mostró que no hubo diferencia estadística entre las desviaciones estándar.

\section{CONCLUSIONES}

Se puede concluir que los residuos de coco y algodón pueden ser utilizados en la fabricación de pellets, los resultados evidencian que pellets elaborados con estas biomasas presentan índice de durabilidad o resistencia a abrasión superior a 97,95\% indicando buena calidad. Los resultados mostraron que no se presentaron aumentos significativos en el PDI para pellets fabricados con aglomerantes (de yuca, plátano o maíz) independientemente de la biomasa utilizada por tanto fue posible producir pellets con buena calidad sin la necesidad de utilizar aglmerante, usando fibra de coco o residuos de algodón con tamaños de partícula inferiores a $2 \mathrm{~mm}$ (10 mesh) y contenido de humedad entre $10 \%$ y $15 \%$.

\section{AGRADECIMIENTOS}

Los autores de este trabajo agradecen al Centro de Investigación para el Desarrollo e Innovación C.I.D.I, a la facultad de Ingeniería Mecánica de la Universidad Pontificia Bolivariana Montería, a la Universidad de Córdoba y al aporte de recursos del convenio 753 de 2013 "Desarrollo de integración tecnológica de recursos energéticos renovables en sistemas productivos agrícolas y agroindustriales Montería, Córdoba, Caribe” BPIN N 2012000100026 (Gobernación de Córdoba-UPB Montería).

\section{REFERENCIAS}

[1] S.C. Bhattacharya. "Biomass Energy and Densification: A global review with emphasis on developing countries". En Proceedings of the First World Conference on Pellets. Stockholm, Sweden. 2002.

[2] L. Wamukonya and B. Jenkins. "Durability and relaxation of sawdust and wheat-straw briquettes as possible fuels for Kenya". Biomass and Bioenergy. Vol. $8 \mathrm{~N}^{\circ} 3$, pp. 175-179. November, 1995. ISSN: 0961-9534. DOI: 10.1016/0961-9534(95)00016-Z.

[3] D.A. Silva, G.T. Nakashima, J.L. Barros, A. L. Da Roz y F. M. Yamaji. "Caracterização de biomassas para a briquetagem". FLORESTA. Vol. $45 \mathrm{~N}^{\circ}$ 4, pp. 713-722. Outubro, 2015. ISSN: 1982-4688. DOI: 10.5380/rf.v45i4.39700.

[4] M.J. Stolarski, S. Szczukowski, J. Tworkowski, M. Krzyżaniak, P. Gulczyński and M. Mleczek. "Comparison of quality and production cost of briquettes made from agricultural and forest origin biomass". Renewable energy. Vol. 57, pp. 20-26. September, 2013. ISSN: 0960-1481. DOI: 10.1016/j.renene.2013.01.005.

[5] F.F. Felfli, J. D. Rocha, D. Filippetto, C. A. Luengo and W. A. Pippo. "Biomass briquetting and its perspectives in Brazil". Biomass and Bioenergy. Vol. $35 \mathrm{~N}^{\circ} 1$, pp. 236-242. January, 2011. ISSN: 0961-9534. DOI: 10.1016/j.biombioe.2010.08.011.

[6] A.K. Tripathi, P.V.R. Iyer and T.C. Kandpal. "A techno-economic evaluation of biomass briquetting in India". Biomass and Bioenergy. Vol. $14 \mathrm{~N}^{\circ}$ 5, pp. 479-488. May-June 1998. ISSN: 0961-9534. DOI: 10.1016/ S0961-9534(97)10023-X.

[7] J. Vinterbäck. "Pellets 2002: the first world conference on pellets". Biomass and Bioenergy. Vol. $27 \mathrm{~N}^{\circ}$ 6, pp. 513-520. December, 2004. ISSN: 0961-9534. DOI: 10.1016/j.biombioe.2004.05.005.

[8] H.S. Kambo and A. Dutta. "Strength, storage, and combustion characteristics of densified lignocellulosic biomass produced via torrefaction and hydrothermal carbonization". Applied Energy. Vol. 135, pp. 182-191. December, 2014. ISSN: 0306-2619.DOI: 10.1016/j.apenergy.2014.08.094

[9] N. Kaliyan and R.V. Morey. "Factors affecting strength and durability of densified biomass products". Biomass and Bioenergy. Vol. 33 $\mathrm{N}^{\circ} 3$, pp.337-359. March, 2009. ISSN: 09619534. DOI: 10.1016/j.biombioe.2008.08.005. 
[10] ASABE. "Cubes, pellets, and crumbles definitions and methods for determining density, durability, and moisture content". ASABE Standards, S269.4, St. Joseph, Mich, USA. pp. 1-8. 2004.

[11] E.R.D. Padilla, I.C.S. Pires, F.M. Yamaji and M. M. Fandiño. "Produção e Caracterização Físico-Mecânica de Briquetes de Fibra de Coco e Palha de Cana-de-Açúcar". Revista Virtual de Química. Vol. 8 N 5, pp. 13341346. Agosto 2016. ISSN: 1984-6835. DOI: 10.21577/1984-6835.20160095.

[12] S. Yaman, M. Şahan, H. Haykiri-Acma, K. Şeşen and S. Küçükbayrak. "Production of fuel briquettes from olive refuse and paper mill waste". Fuel Processing Technology. Vol. 68 $\mathrm{N}^{\circ}$ 1, pp. 23-31. October, 2000. ISSN: 03783820. DOI: $10.1016 / \mathrm{S} 0378-3820(00) 00111-9$.

[13] I. Obernberger and G. Thek. "Physical characterization and chemical composition of densified biomass fuels with regard to their combustion behavior". Biomass and Bioenergy. Vol. $27 \mathrm{~N}^{\circ}$ 6, pp. 653-669. December, 2004. ISSN: 0961-9534. DOI: 10.1016/j.biombioe.2003.07.006.

[14] DANE, "Encuesta Nacional Agropecuaria". 24/01/2018. URL: http://goo.gl/qfWgqi

[15] MinCIT, "Perfil económico: Departamento de Córdoba, año base 2013", Oficina de Estudios Económicos Montería, 2013, 24/01/2018. URL: http://goo.gl/gLHDxU

[16] ASTM. "Standard specification for woven wire test sieve cloth and test sieves". ASTM Standards, E11 - 13, West Conshohocken, PA, USA. pp. 1-9. 2013. DOI: 10.1520/E0011.

[17] D.C. Montgomery. "Design and Analysis of Experiments". John Wiley and Sons. 7nd. Ed. New York, USA. pp. 455. 2008. ISBN: 968-18-6156-6.

[18] I. Obernberger and G. Thek. "The Pellet Handbook - The Production and Thermal Utilisation of Biomass Pellets". Taylor \& Francis Group. 1st ed, London, UK. pp. 57. 2010. ISBN: 978-1-84407-631-4.

[19] D. Andreiko and J. Grochowicz. "Effect of the moisture content on compression energy and strength characteristic of lupine briquettes". Journal of Food Engineering. Vol. $83 \mathrm{~N}^{\circ} 1$, pp. 116-120. November, 2007. ISSN: 02608774. DOI: 10.1016/j.jfoodeng.2006.12.019. [20] W. Stelte, J. K. Holm, A. R. Sanadi, J. Ahrenfeldt and U. B Henriksen. "Fuel pellets from biomass: The importance of the pelletizing pressure and its dependency on the processing conditions". Fuel. Vol. $90 \mathrm{~N}^{\circ} 11$, pp. 3285-3290. November, 2011. ISSN: 0016-2361. DOI: doi.org/10.1016/j. fuel.2011.05.011.

[21] T. Filbakk, G. Skjevrak, O. Hoibo, J. Dibdiakova and R. Jirjis. "The influence of storage and drying methods for scots pine raw material on mechanical pellet properties and production parameters". Fuel Processing Technology. Vol. $92 \mathrm{~N}^{\circ}$ 5, pp. 871-878. May, 2011. ISSN: 0378-3820. DOI: 10.1016/j. fuproc.2010.12.001.

[22] M.T. Carone, A. Pantaleo and A. Pellerano. "Influence of process parameters and biomass characteristics on the durability of pellets from the pruning residues of Olea europaea L". Biomass and Bioenergy. Vol. $35 \mathrm{~N}^{\circ} 1$, pp. 402-410. January, 2011. ISSN: 0961-9534. DOI: 10.1016/j.biombioe.2010.08.052.

[23] W. Stelte, A. R. Sanadi, L. Shang, J. K. Holm, J. Ahrenfeldt and U. B. Henriksen. "Recent developments in biomass pelletization-A review". BioResources. Vol. $7 \mathrm{~N}^{\circ} 3$, pp. 4451-4490. August 2012. ISSN: 1930-2126. URL: https://goo.gl/KfiZx3.

[24] N. Arzola, A. Gómez and S. Rincón. "The effects of moisture content, particle size and binding agent content on oil palm shell pellet quality parameters". Ingeniería e Investigación. Vol. $32 \mathrm{~N}^{\circ}$ 1, pp. 24-29. April, 2012. ISSN: 0120-5609. URL: http://www. scielo.org.co/pdf/iei/v32n1/v32n1a05.pdf

[25] S. Yaman, M. Sahanşahan, H. HaykiriAcma, K. Seșen and S. Küçükbayrak. "Fuel briquettes from biomass-lignite blends". Fuel processing technology. Vol. $72 \mathrm{~N}^{\circ} 1$, pp.1-8. August, 2001. ISSN: 0378-3820. DOI: 10.1016/S0378-3820(01)00170-9. 